\title{
Review Article \\ Systematic Review of Basic Research on Alzheimer's Disease with Shen Zhi Ling Oral Liquid
}

\author{
Yahan Wang, 1 Chunxiang Liu $\mathbb{D},{ }^{2}$ Hui Wang $\mathbb{D},{ }^{2}$ Yin Jiang, \\ Pengwen Wang $\left(\mathbb{D},{ }^{1}\right.$ and Hongcai Shang $\mathbb{C}^{1,3}$ \\ ${ }^{1}$ Key Laboratory of Chinese Internal Medicine of Ministry of Education and Beijing, Dongzhimen Hospital, \\ Beijing University of Chinese Medicine, Haiyuncang Lane, Dongcheng District, Beijing 100700, China \\ ${ }^{2}$ Evidence-Based Medicine Center, Tianjin University of Chinese Medicine, Beihua South Road, Jinghai District, Tianjin 301617, China \\ ${ }^{3}$ Center for Evidence-Based and Translational Medicine, Jiangxi University of Traditional Chinese Medicine, Wanli District, \\ Nanchang, Jiangxi 330000, China
}

Correspondence should be addressed to Pengwen Wang; pw_wang@163.com and Hongcai Shang; shanghongcai@126.com

Received 22 November 2018; Revised 28 February 2019; Accepted 18 March 2019; Published 21 April 2019

Academic Editor: Dolores García Giménez

Copyright (C) 2019 Yahan Wang et al. This is an open access article distributed under the Creative Commons Attribution License, which permits unrestricted use, distribution, and reproduction in any medium, provided the original work is properly cited.

Objective. The present report systematically reviewed the basic research of Shen Zhi Ling oral liquid (Tiao Xin preparation) treatment on Alzheimer's disease (AD). Methods. CNKI, Wanfang, and VIP were searched, and the literature was selected according to inclusion and exclusion criteria. Data were extracted, and descriptive analysis was used. Results. Twenty-four articles were included, all of which were published as "Tiao Xin preparation." There were seven types of AD models involved. The mechanism of action of Shen Zhi Ling oral liquid in the treatment of AD primarily included suppression of A $\beta$ deposition and tau hyperphosphorylation, regulation of multiple neurotransmitters, improvement in energy metabolism, and promotion of the expression of autophagy-related and learning-memory-associated proteins. Conclusions. AD is a complex disease caused by multiple factors. Shen Zhi Ling oral liquid exhibited multiple and multitarget effects and great potential for treating AD. The continuous development of molecular biology and related disciplines will further elucidate the mechanism of Shen Zhi Ling oral liquid intervention in $\mathrm{AD}$.

\section{Introduction}

Dementia is a chronic progressive disease that is primarily characterized by intelligence impairments. These properties enable the disease to hide and progress slowly. The prevalence of dementia increases annually. The WHO predicts 29 million dementia patients worldwide by 2020,2/3 of whom will suffer from Alzheimer's disease (AD) [1]. AD is roughly divided into early, middle, and late stages based on cognitive severity, and the clinical proportion of each stage is approximately 4:3:3 [2]. Elderly patients in the late stage lose their ability to care for themselves, behave abnormally, and exhibit low intelligence and memory loss. The poor clinical outcomes of patients with dementia in the middle and late stages cause serious mental and economic burdens on families and society. Therefore, early intervention in patients with $\mathrm{AD}$ or high-risk groups is essential.
Shen Zhi Ling oral liquid (also known as Shen Gui Jian Nao oral liquid, Yang Xin Jian Nao oral liquid, Nao Rui Kang oral liquid, and Tiao Xin preparation) is the first new traditional Chinese medicine compound drug in China to be approved by the China Food and Drug Administration (CFDA) (Z20120010) for the treatment of mild-to-moderate AD. This compound consists of Codonopsis pilosula, Cinnamomi mulus, Paeonia lactiflora, Radix Glycyrrhizae Preparata, Poria, ginger, Polygala, Grassleaf Sweetflag Rhizome, Fossilia Ossia Mastodi, and common oyster shell. Shen Zhi Ling oral liquid enriches qi, warms yang, reduces phlegm, and soothes the nerves, and it is suitable for patients with heart-qi deficiency syndrome.

Professor Lin Shuimiao of the Shanghai Geriatric Institute of Chinese Medicine prescribed the oral liquid based on the formulas of "Kai Xin Powder" and "Ling Ren Bu Wang (unforgettable formula)" in Handbook of Prescriptions 
for Emergencies, which consists of ginseng, Poria cocos, Polygala root, and Calamus under the theory of Heart Dominating Spirit and Mind. Previous studies confirmed that Shen Zhi Ling oral liquid treats $\mathrm{AD}$ via the regulation of multiple central neurotransmitters, the inhibition of neuronal apoptosis and injury, and inhibition of hyperphosphorylation of tau protein [3]. The present article systematically reviews the basic research of AD treatment with Shen Zhi Ling oral liquid, analyzes the progress of research on its mechanism of action, and provides baseline data for further research and development.

\section{Materials and Methods}

2.1. Research Objective. This review is a basic study of Shen Zhi Ling oral liquid for AD treatment and document analyses of its mechanism of action.

2.2. Search Method. To ensure a full recall of the appropriated studies, "Shen Zhi Ling oral liquid," "Shen Gui Jian Nao oral liquid," "Yang Yin Jian Nao liquid," "brain Ruikang oral liquid," “Tiaoxin fang," “Tiaoxin recipe," or "Tiaoxin prescription" were used as search words. The following databases were searched: China Knowledge Network "China Academic Journals Network Publishing Bank" (1979 11/30/2016), Chongqing Web Information Co., Ltd. "Chinese Science and Technology Journal Database" (1989 2016), Beijing Wanfang Data Co., Ltd. "Million Party Data Resource System (1998 2016), and PubMed (until 11/30/2016).

2.3. Inclusion Criteria. Basic research studies of Shen Zhi Ling oral liquid use in $\mathrm{AD}$ rodent models were included.

2.4. Exclusion Criteria. Duplicate publications, clinical studies, reviews or other irrelevant publications, studies with the same drug name but different ingredients, basic research using non-AD disease models, split or effective site experimental studies, incomplete description of disease models or mechanism of action, and repeated publications of the same study were excluded.

2.5. Data Acquisition and Analysis. Excel was used to create a database, and retrieved articles were read one at a time. Relevant data were entered, managed, and analyzed. Descriptive analysis was used to examine the mechanism of action of Shen Zhi Ling oral liquid on AD.

\section{Results}

3.1. Literature Search and Filter Results. A total of 395 articles were initially screened out, and the remaining articles were carefully read according to the inclusion and exclusion criteria. Twenty-four articles were ultimately incorporated into the analysis. The document screening procedure is presented in Figure 1.

3.2. Literature Overview. The 24 articles included were published from 1998 to 2016. Most of the articles were the research of Professor Lin's team from Shanghai University of Traditional Chinese Medicine, and all of the articles were published under the name of the "Tiao Xin preparation." These articles used eight AD models: SAMP8 mice, APP/PS1 double-transgenic mice, $\mathrm{A} \beta_{1-40}$ fragment left-lateral ventricle injection, $\mathrm{A} \beta_{25-35}$ unilateral amygdala injection, dihydroxyfumarate (DHF) and ferric chloride-adenosine diphosphate $\left(\mathrm{FeCl}_{3}-\mathrm{ADP}\right)$ left ventricle injection, oxidative damage due to reactive oxygen species, unilateral electrolytic lesion of the forebrain basal ganglia, and the combination of D-galactose subcutaneous injection and amanitin acid brain injection [428]. The details are shown in Table 1.

3.3. Analysis of Shen Zhi Ling Oral Liquid (Tiao Xin Preparation) on AD Rodent Models. The experiments in the literature used different rodent models, but studies on the mechanism of the drug were not affected. Tiao Xin preparation in the treatment of $\mathrm{AD}$ primarily included the following mechanisms of action.

3.3.1. Inhibition of $A \beta$ Deposition and Hyperphosphorylation of Tau Protein. The two major pathological features of AD are senile plaques (SPs), with $\mathrm{A} \beta$ serving as the main component, and neurofibrillary tangles (NFTs) being formed by hyperphosphorylated tau protein. $\mathrm{A} \beta$ deposition activates glial cells to secrete a variety of inflammatory cytokines and induces inflammatory responses, which further promote the expression of APP and mRNA and the deposition of $\mathrm{A} \beta$, and initiates inflammation and immune cascades to induce the formation of SPs. This study found that the severity of dementia symptoms in AD patients positively correlated with the number of NFTs in brain tissue. The abnormal hyperphosphorylation of tau protein leads to the loss of the normal biological activity of catalytic microtubule assembly and stabilization of microtubule structure, and it becomes a cytotoxic molecule that promotes its own deposition as NFTs [3]. A $\beta$ activates multiple protein kinases that overly phosphorylate tau protein to PHF-tau protein and form NFTs.

Hui Zhou et al. showed that the Tiao Xin preparation effectively inhibited the increase in the mRNA of cytokines IL- $1 \beta$ and IL- 6 and APP in AD model mice [4]. Liu Xueyuan et al. confirmed that the Tiao Xin preparation reduced the expression of GFAP and APP mRNA (751/770) in AD models, which inhibited the activation of glial cells and reduced inflammatory reactions and the large amount of amyloid$\beta$ protein deposition to reduce the formation of SPs [5]. Sun et al. found that the Tiao Xin preparation reduced $\mathrm{APP}$ gene expression in $\mathrm{AD}$ brains and $\mathrm{A} \beta$ deposition by decreasing the effects of active oxygen on NF- $\kappa \mathrm{B}$ [6]. Hong Daojun et al. found that the Tiao Xin preparation inhibited the expression of phosphorylated tau protein, $\mathrm{A} \beta$ protein, P35 protein, and cell cycle-related proteins (cyclinA and cyclinB1) in brain $[7,8]$. Zhao Weikang et al. showed that the Tiao Xin preparation inhibited the expression of tau protein phosphorylation kinases (GSK-3 $\beta$ and P38 MAPK) and abnormally phosphorylated tau protein (AT-8), which reduced NFTs [9]. 


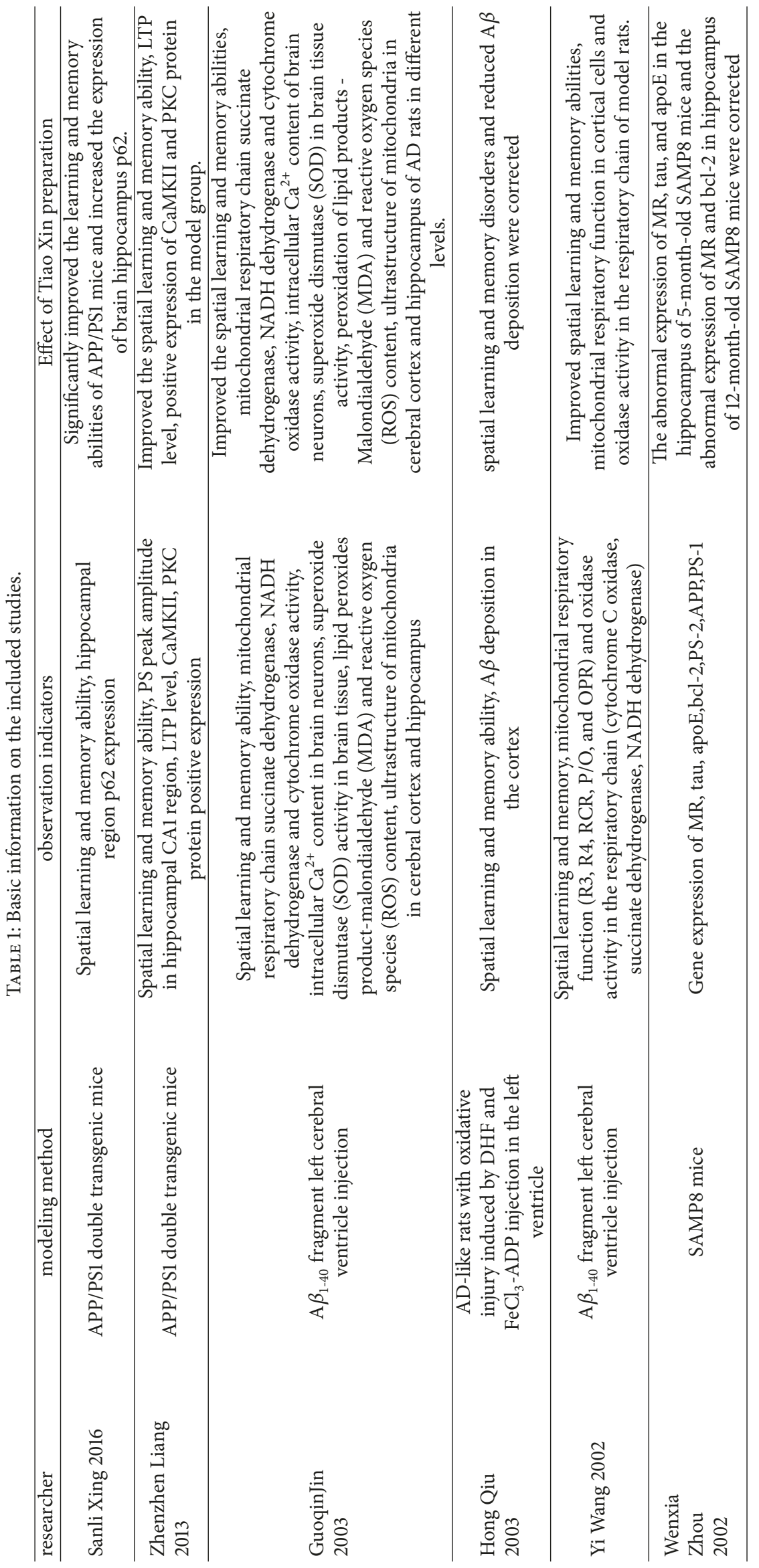




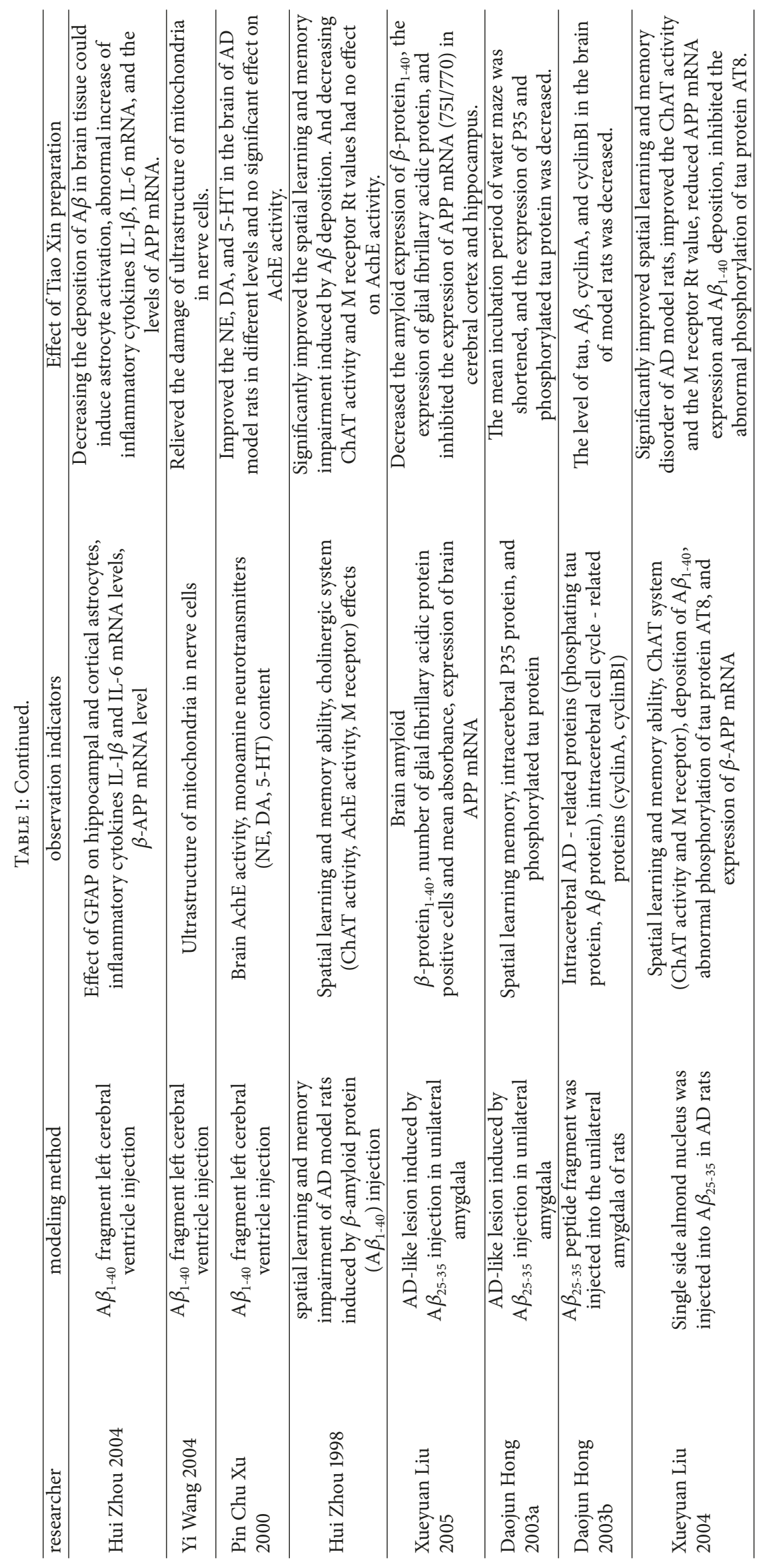




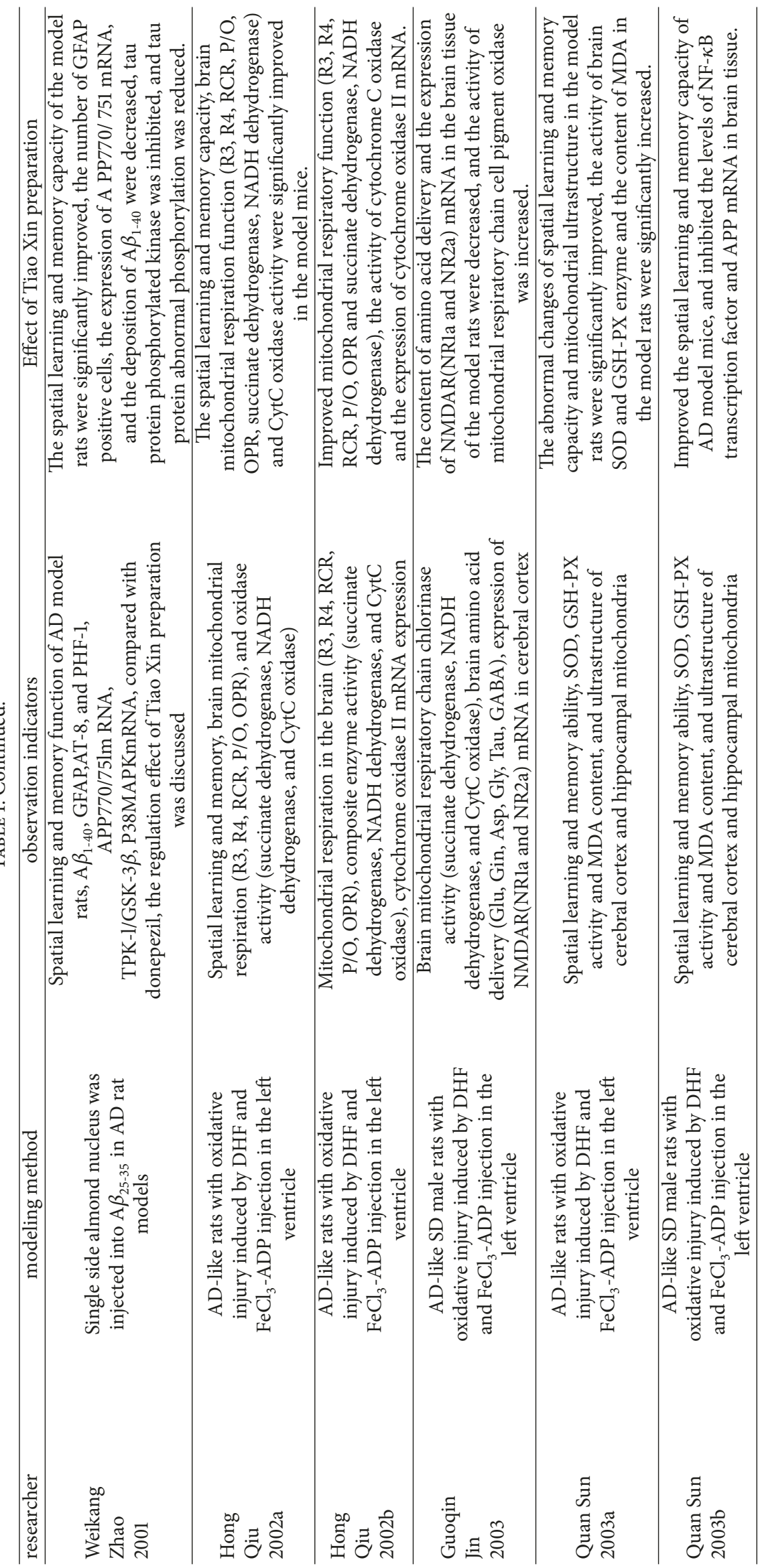




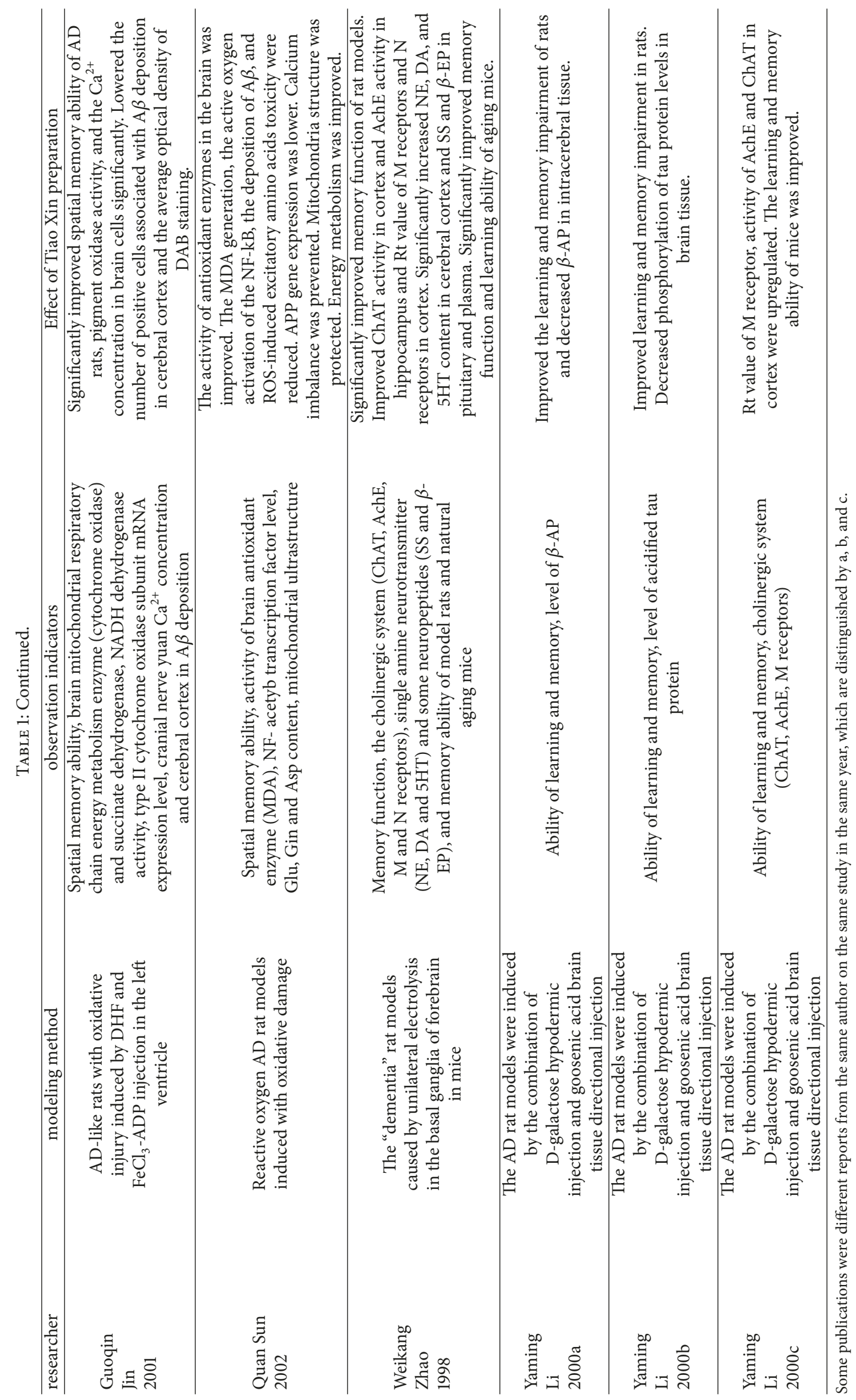




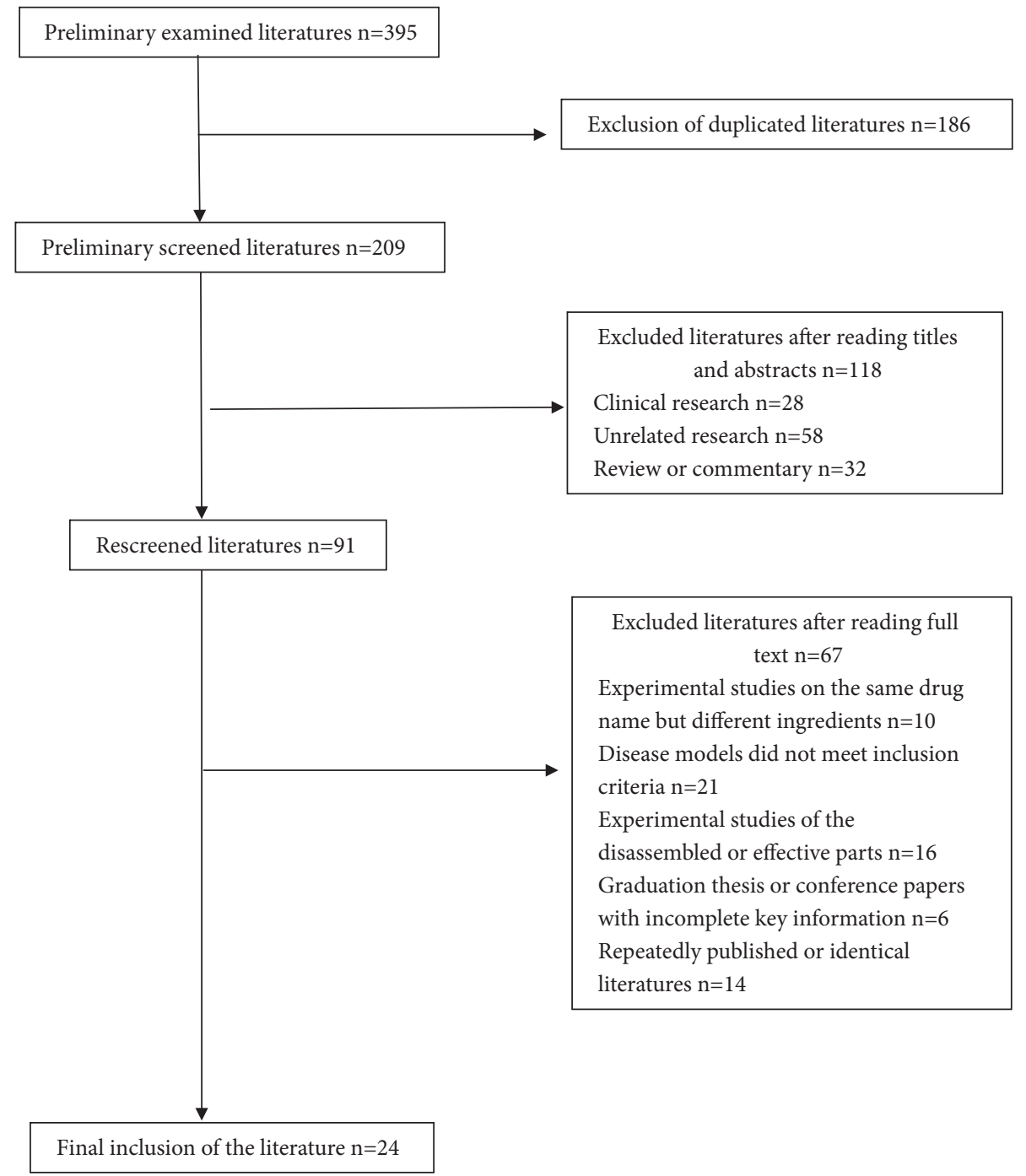

FIGURE 1: Document screening flow chart.

3.3.2. Multiple Neurotransmitter Regulation. The central cholinergic system and monoamine transmitters (NE, DA, and 5-HT) are closely related to learning and memory. The central cholinergic system primarily excites the central nervous system by regulating the transition from first-level memory to second-level memory processes through two pathways, the septal-hippocampal-peripheral leaf and the cerebral cortex. Choline acetyltransferase (ChAT) is a key enzyme in the synthesis of human acetylcholine (ACh), and it is an important marker of cholinergic activity. ChAT activity is significantly reduced primarily due to cortical postsynaptic neuron degeneration, which leads to the degenerative changes of cholinergic neurons in the basal ganglia of $\mathrm{AD}$ patients' brains from the basal ganglia to the cortex [3]. Recent studies found that NMDARs and its subunits NR2A and NR2B were involved in the development of the central nervous system and the formation of learning and memory, and the distribution and expression of these receptors was closely related to the occurrence and development of cognitive disorders [10]. The Tiao Xin preparation increased ChAT activity in the cortex, AchE in the hippocampus, and the Rt values of $M$ receptors and the cortical $N$ receptors in AD models. This preparation also increased NE, DA, and $5 \mathrm{HT}$ in the cerebral cortex and SS and $\beta$-EP levels in the pituitary and plasma of $\mathrm{AD}$ models [11]. JinGuoqin et al. demonstrated that the Tiao Xin preparation reduced amino acid transmitter content (excitogenic amino acid transmitters (Glu, Gln, and Asp) and inhibitory amino acid transmitters (Gly, Tau, and GABA)) and the expression of NMDAR mRNA (NR1a and NR2a) for AD prevention and treatment [12].

3.3.3. Energy Metabolism Improvement. Brain tissue is rich in mitochondria, and it is an important site for cellular respiration and energy metabolism. Brain tissue is also the main site for the production of oxygen free radicals. Impairment of mitochondrial respiratory function or respiratory chain complex enzyme activity creates disorder in brain energy 
metabolism. A large number of free radicals may damage mitochondrial structure and function, which decreases enzyme activity, endangers the energy metabolism mechanism, and affects the function of the cholinergic neurons that cause progressive learning and memory impairment in $\mathrm{AD}$ patients. Mitochondrial respiration III state (R3) is the rate of oxygen consumption during the fast oxygen consumption period when the substrate ADP is added. Respiration state IV (R4) is the oxygen consumption rate after ADP exhaustion in state III. The ratio of R3 and R4 is the respiratory control rate (RCR). P/O and OPR indicate the efficiency of synthesizing ATP via the mitochondrial respiratory chain releasing energy to couple ADP.

Qiu Hong et al. demonstrated that mitochondrial respiratory function (R3, R4, RCR, P/O, and OPR), complex enzyme activities (succinate dehydrogenase, NADH dehydrogenase, and CytC oxidase), and the expression of cytochrome oxidase II subunit mRNA were significantly reduced in cerebral tissue from AD model, and the Tiao Xin preparation significantly improved these indicators [13]. Sun Quan et al. showed that the activities of SOD and GSH-PX in the cortex and hippocampal subregions decreased significantly in $\mathrm{AD}$ models, which indicates a decreased ability of free radical scavenging in brain tissue [14]. The reason for this decrease is likely that too much reactive oxygen species (ROS) damages the oxidation of protein structures, which may reduce or inactivate enzyme activity and significantly increase malondialdehyde (MDA) content in the cerebral cortex and hippocampus. MDA is a product of the lipid peroxidation of the polyunsaturated fatty acids that are produced in ROS-attacking biofilms. The amount of MDA may reflect the degree of lipid peroxidation in the body and indirectly reflect the extent of cell damage. The abnormal changes of mitochondria in the cortex and hippocampus of rats suggest that reactive oxygen damages the ultrastructure of mitochondria. The Tiao Xin preparation increased SOD and GSH-PX enzyme activity and reduced MDA content to improve energy metabolism, protect mitochondrial structure from damage, and prevent the occurrence and development of $\mathrm{AD}$. JinGuoqin et al. showed that the concentration of calcium ions in cortical and hippocampal neurons increased abnormally in AD models [15]. The overload of $\mathrm{Ca}^{2+}$ could further promote the lipid peroxidation and free radical formation that aggravate $\mathrm{AD}$ pathogenesis. The Tiao Xin preparation reduced the $\mathrm{Ca}^{2+}$ concentration in the cytoplasm of cortical and hippocampal neurons and improved calcium homeostasis in neurons of $\mathrm{AD}$ rats, which is conducive to maintaining neuronal structure and function.

\subsubsection{Research on Other Memory-Related Mechanism} Improvement. Recent studies found that defects in autophagy may be closely related to AD occurrence and development. Model mice with removed autophagy-related genes exhibit typical neurodegenerative changes, which indicate a key role of autophagy in neurodegenerative diseases. Autophagy is the intracellular lysosome-mediated degradation of abnormally damaged proteins and organelles, and it plays an important role in maintaining homeostasis of the cell environment. Therefore, the removal of abnormally accumulated proteins in neurons via autophagy is important to maintain normal neurons. The P62 protein is a linker protein that combines with ubiquitinated proteins as a selective substrate for autophagy, and it reflects cell autophagic activity. Sanli Xing et al. showed that the Tiao Xin preparation upregulated the expression of autophagy-related protein P62 in $\mathrm{AD}$ models, promoted the activation of autophagy, and improved learning and memory abilities [16]. Zhenzhen Liang noticed that the Tiao Xin preparation induced the expression of learning and memory-related proteins (CaMKII and PKC) and improved the inhibition of long-term potentiation (LTP) in hippocampus [17].

\section{Discussion}

The pathological mechanism of $\mathrm{AD}$ is very complex, and it has not been fully elucidated to date, which hinders $\mathrm{AD}$ prevention and treatment research. The FDA has only approved 5 drugs for sale, and clinical studies on anti-AD drugs have been unsuccessful for a long time [29]. Basic research supports various hypotheses that the pathogenesis of $\mathrm{AD}$ is primarily related to the excessive deposition of amyloid protein, phosphorylation of tau protein, the mitochondrial cascade, and neuroinflammation, rather than an inevitable outcome of brain aging [30]. These studies overturned the previous understanding of the cause of $\mathrm{AD}$ that amyloid$\beta$ is good for health, and it is synthesized by the body to eliminate harmful molecules related to inflammation and immune responses, and it relieves the symptoms of AD and reverses neurodegenerative diseases, such as $\mathrm{AD}$ [31].

This study summarized the historical basic research on Shen Zhi Ling oral liquid treatment of $\mathrm{AD}$ and revealed the following main mechanisms: (1) inhibition of the excessive deposition of amyloid protein and phosphorylation of tau protein; (2) regulation of multiple neurotransmitters; (3) improving energy metabolism; and (4) improving the expression of autophagy-related proteins and learning-memoryassociated proteins. An increasing number of scholars believe that the key to $\mathrm{AD}$ treatment lies in the early stage, especially during the symptomatic prodrome. Studies showed that the Shen Zhi Ling oral liquid regulated the expression of some AD-related genes in the hippocampus and cortex of rapidly aging mice, improved cognitive function via the removal of abnormal proteins, protected normal synaptic transmission, and regulated signal transduction and other molecular pathways [32].

There is a new Chinese medicine compound patent HTJDT-M (US patent No. 9,375,457), in which Rhizoma coptidis (RC), Cortex phellodendri (CP), and Fructus gardeniae (FG) are prepared in a dry weight ratio of 2:2:3. According to animal studies, HTJDT-M could improve the cognitive dysfunction of 3XTg-AD mice by reducing $\mathrm{A} \beta$ deposition, decreasing the level of detergent soluble and acid-soluble $\mathrm{A} \beta$ via decreasing the levels of full length amyloid- $\beta$ precursor protein and C-terminal fragments of APP $[33,34]$. In addition, it can relieve cognitive impairment caused by cerebral ischemia and disturbance of the cholinergic system $[33,35]$. At present, most traditional Chinese medicines for $\mathrm{AD}$ are used as formulas. In addition to insomnia, diabetes, and 
glomerulonephritis, Liuwei Dihuang decoction and Kamiondam-tang (KOT) could improve cognitive impairment by promoting hippocampal neurogenesis in adult rats [36, 37]. KOT can activate p-CREB, p-Akt, BDNF, and other memory-related proteins to improve the spatial memory disorder of AD [38]. Kami-shoyo-san and Danggui-ShaoyaoSan treat $\mathrm{AD}$ by dispersing the depressed liver energy and have neuroprotective effects, which can reverse the cognitive degradation of aging mice and maintain the cerebral cortex structure [39]. Another traditional Chinese medicine formula, Fu Zheng San (FZS), consists of ginseng, radix scutellariae, calamus root, and liquorice. SAMP8 mice were used to study its effect on promoting neurogenesis. However, there is still a lack of observation on the role of this drug in the early pathological changes of AD [40]. Studies on Shen Zhi Ling oral liquid based on different kinds of mouse or rat models have demonstrated its effectiveness in the complex pathological manifestations of $\mathrm{AD}$, such as $\mathrm{A} \beta$ deposition, nerve fiber tangles, synaptic plasticity abnormalities, myelin injury, and neuroinflammatory responses, which are more comprehensive and in depth.

$\mathrm{AD}$ is a complex disease caused by multiple factors, which underlies the difficulty of treatment with monomeric drugs. Chinese medicine exerts multiple effects and multiple mechanisms of action, which may produce unexpected effects for $\mathrm{AD}$ treatment. Treatment for $\mathrm{AD}$ requires considerably more research, but developments in molecular biology and its related disciplines provide an opportunity to further elucidate the pathogenesis of $\mathrm{AD}$.

\section{Abbreviations}

$\begin{array}{ll}\text { A } \beta: & \text { Amyloid } \beta \\ \text { GFAP: } & \text { Fibrillary acid protein } \\ \text { Il-1 } \beta: & \text { Interleukin }-1 \beta \\ \text { Il-6: } & \text { Interleukin-6 } \\ \beta \text {-APP mRNA: } & \text { Amyloid protein precursor protein gene } \\ \text { AchE: } & \text { Acetylcholinesterase } \\ \text { NE: } & \text { Norepinephrine } \\ \text { DA: } & \text { Dopamine } \\ \text { 5-ht: } & \text { 5-Hydroxytryptamine } \\ \text { ChAT: } & \text { Acetylcholine transferase } \\ \text { Glu: } & \text { Glutamate } \\ \text { Gin: } & \text { Glutamine } \\ \text { Asp: } & \text { Aspartic acid } \\ \text { Gly: } & \text { Glycine } \\ \text { Tau: } & \text { Taurine } \\ \text { GABA: } & \text { Constant-aminobutyric acid } \\ \text { SOD: } & \text { Superoxide dismutase } \\ \text { GSH - PX: } & \text { Glutathione peroxidase } \\ \text { MDA: } & \text { Malondialdehyde } \\ \text { SS: } & \text { Somatostatin } \\ \beta \text {-EP: } & \text { Abolitionists - endorphins } \\ \text { NMDAR: } & \text { n-methyl d-aspartic acid receptor } \\ \text { MR: } & \text { Mineralocorticoid receptor } \\ \text { Apo E: } & \text { Apolipoprotein E } \\ \text { Bcl-2: } & \text { B-cell lymphoma-2 } \\ \text { PS1: } & \text { Presenilin-1 } \\ \text { PS2: } & \text { Presenilin-2 }\end{array}$

p-CREB: Phosphorylated cyclase response element binding protein

p-Akt: $\quad$ Protein kinase B

BDNF: Brain derived neurotrophic factor.

\section{Conflicts of Interest}

The authors declare that there are no conflicts of interest regarding the publication of this paper.

\section{Authors' Contributions}

Chunxiang Liu, Hui Wang, Yahan Wang, and Yin Jiang are cofirst authors.

\section{References}

[1] C. Dufouil and A. Alperovitch, "Epidemiology of Alzheimer's disease," La Revue du Praticien, vol. 55, no. 17, pp. 1869-1878, 2005.

[2] L. F. Shen, "Advances in Alzheimer's disease research," Modern Medical Hygiene, vol. 26, no. 4, pp. 542-543, 2010.

[3] S. Jingli, Z. Yonghua, and W. Weikang, "Study on the mechanism ofTiao Xin preparation in the treatment of senile dementia," Jilin Journal of Traditional Chinese Medicine, vol. 28, no. 3, pp. 211-212, 2008.

[4] H. Zhou, W. K. Zhao, and G. Q. Jin, "Effects of TiaoXin decoction on the expression of Il-1 $\beta$, Il- 6 and APPmRNA in the brain tissue of the rat model of AD," Chinese Journal of Integrative Chinese and Western Medicine, vol. 24, no. 6, pp. 599533, 2004.

[5] X. Y. Liu, W. K. Zhao, P. C. Xu, and S. M. Lin, "Effect of TiaoXin preparation on APP mRNA expression in rat model of dementia induced by Aß25 35 amygdaloid injection," Journal of Chinese Clinical Rehabilitation, vol. 9, no. 28, pp. 130-132, 2005.

[6] Q. Sun, Study on the Toxic Effect of Reactive Oxygen on Rat Brain and the Adjustment Mechanism of Cardiac Regulation, Shanghai University of Traditional Chinese Medicine, 2002.

[7] D. J. Hong, A. L. Pei, S. D. Lu et al., "Effects of TiaoXin decoction on the phosphorylation of P35 and tau protein by means of $\mathrm{A} \beta_{25 \sim 35}$ amygdaloid injectioninrats," Chinese Journal of Geriatrics, vol. 22, no. 11, pp. 673-676, 2003.

[8] D. J. Hong, A. L. Pei, and Z. Q. Zhu, "Effects of TiaoXin decoction on the expression of cell cycle-related proteins in the brain of rats injected with $\mathrm{A} \beta_{25 \sim 35}$," Chinese Journal of Basic Medicine of Traditional Chinese Medicine, vol. 9, no. 7, pp. 58-60, 2003.

[9] W. K. Zhao, X. Y. Liu, and P. C. Xu, “The change of Tau protein phosphorylation and APPmRNA in A $\beta$-induced rat models and effects of TiaoXin recipe," in Proceedings of the Chinese Society of Biochemistry and Molecular Biology of the Eighth Member Congress and Academic Conferences, Shanghai, China, 2001.

[10] X. Wang, Y. Huang, F. Wang, and Y. Zou, "Association of NMDAR, NR2A, and NR2B with cognitive impairment in diabetic patients," Journal of Southern Medical University, vol. 33, no. 12, pp. 1848-1851, 2013.

[11] W. K. Zhao, X. D. Dai, and S. J. ShenTu, "Study on the effects of TiaoXin formula on animal models of dementia and memory impairment," Journal of Shanghai Institute of Traditional Chinese 
Medicine, Shanghai University of Traditional Chinese Medicine, vol. 12, no. 2, pp. 59-62, 1998.

[12] G. Q. Jin, H. Qiu, Q. Sun et al., "Effects of TiaoXin decoction on the activity of respiratory chain oxidase and the content of amino acid delivery in brain tissue of AD rats," Journal of Shanghai University of Traditional Chinese Medicine, vol. 17, no. 3, pp. 39-41, 2003.

[13] H. Qiu, G. Q. Jin, X. L. Zhang et al., "Effects of TiaoXin recipe on energy metabolism of AD rats with oxidative injury," Pharmacology and Clinical Practice of Traditional Chinese Medicine, vol. 18, no. 1, pp. 18-21, 2002.

[14] Q. Sun, G. Q. Jin, W. K. Zhao et al., "Effects of TiaoXin decoction on antioxidant capacity of brain tissue in AD rats," Chinese Medicine Science And Technology, vol. 10, no. 5, pp. 273-274, 2003.

[15] G. Q. Jin, Y. Wang, X. L. Zhang et al., "Experimental study on the effect of Tiao Xin recipe on the "harmful network" of AD rats induced by $\mathrm{A} \beta$," Pharmacology and Clinic of Traditional Chinese Medicine, vol. 19, no. 3, pp. 20-23, 2003.

[16] S. L. Xing, D. Z. Shen, C. Chen et al., "Effects ofTiao Xin preparation on learning and memory ability and expression level of p62 in AD mice," Chinese Medicine Acute Diseases, vol. 25, no. 1, pp. 45-47, 2016.

[17] Z. Z. Liang, Experimental Study on Intervention of Cardiac Intervention in Early Cognitive Impairment of Alzheimer's Disease, Shanghai University of Traditional Chinese Medicine, 2013.

[18] X. Y. Liu, W. K. Zhao, P. C. Xu, and S. M. Lin, "Effects of Tiao Xin decoction on the model rat of AD induced by amygdaloid injection of A $\beta 25 \sim 35$," Chinese Herbal Medicine, vol. 35, no. 1, pp. 50-54, 2004.

[19] Y. Wang, G. Q. Jin, W. R. Xu, and X. M. Ye, "Effects of TiaoXin decoction on the ultrastructure of nerve cells in AD rats," Advances in Anatomical Science, vol. 10, no. 57, 2004.

[20] Q. Sun, G. Q. Jin, W. K. Zhao et al., "Effects of TiaoXin decoction on the expression of amyloid protein (APP) mRNA in brain tissues of rats with oxidative injury AD," Pharmacology and Clinical Practice of Traditional Chinese Medicine, vol. 19, no. 2, pp. 28-31, 2003.

[21] Y. Wang, G. Q. Jin, W. R. Xu et al., "Effects of TiaoXin recipe on mitochondrial respiration function of $\mathrm{AD}$ rats caused by $\mathrm{A} \beta$," Chinese Journal of Gerontology, vol. 22, no. 3, pp. 206-208, 2002.

[22] H. Qiu, G. Q. Jin, W. K. Zhao et al., "Effects of TiaoXin recipe on spatial memory and mitochondrial respiratory function in oxidative damaged AD rats," Chinese Journal of Basic Medicine of Traditional Chinese Medicine, vol. 8, no. 6, pp. 26-29, 2002.

[23] G. Q. Jin, W. K. Zhao, H. Qiu, X. L. Zhang, and S. M. Lin, "Effects of TiaoXin recipe on the "harmful network" in AD rats with oxidative damage," in Proceedings of the Chinese Society of Biochemistry and Molecular Biology of the Eighth Member Congress and Academic Conference, Shanghai, China, 2001.

[24] Y. M. Li, C. Y. Zhang, and S. M. Lin, "Experimental study on the effects of TiaoXin recipe on learning memory and cholinergic system of dementia rats induced by $\mathrm{D}$-galactose combined with goosenic acid," Chinese Medicine Science And Technology, vol. 7, no. 4, pp. 237-238, 2000.

[25] Y. M. Li, C. Y. Zhang, and S. M. Lin, "Effects of TiaoXin recipeon learning and memory of $\mathrm{AD}$ rats caused by $\mathrm{D}$ galactose combined with goosenic acid," Gansu Traditional Chinese Medicine, vol. 3, pp. 59-61, 2000.

[26] Y. M. Li, C. Y. Zhang, and S. M. Lin, "Effects of TiaoXin formula on learning memory and brain phosphorylation of tau protein in dementia rats induced by $\mathrm{D}$-galactose combined with goose cream qin acid," Heilongjiang Chinese medicine, vol. 03, pp. 5253, 2000.

[27] P. C. Xu, S. M. Lin, W. K. Zhao, Z. B. Yin, and S. W. Chen, "Effects of drugs nourishing heart and kidney on brain acetylcholinesterase and monoamine neurotransmitters in $\mathrm{AD}$ rats," Journal of Chinese Medicine, vol. 15, pp. 344-346, 2000.

[28] H. Zhou and W. K. Zhao, "Effects of Tiao Xin formula on spatial learning and memory impairment and cholinergic energy system in rat models of dementia," Pharmacology and Clinical Practice of Traditional Chinese Medicine, vol. 14, no. 3, pp. 2931, 1998.

[29] H. Li, "Clinical research progress in drug treatment of Alzheimer's disease," Chinese Journal of New Drugs, vol. 26, no. 6, pp. 648-655, 2017.

[30] H. Du and X. D. Yuan, "Research progress of etiology and pathogenesis of Alzheimer's disease," Journal of Shan Dong University (Medical Edition), vol. 55, no. 10, pp. 21-27, 2017.

[31] M. P. Kurnellas, C. M. Adams, R. A. Sobel, L. Steinman, and J. B. Rothbard, "Amyloid fibrils composed of hexameric peptides attenuate neuroinflammation," Science Translational Medicine, vol. 5, no. 179, pp. 1-20, 2013.

[32] X. R. Cheng, J. Huang, Y. Zheng, W. X. Zhou, and Y. X. Zhang, "Effects of Tiao Xin formula on gene expression in hippocampus and cortex of rapid aging mice," in Proceedings of the Abstract of the Paper of the 11th National Conference on Traditional Chinese Medicine Pharmacology, 2010.

[33] S. S. K. Durairajan, L.-F. Liu, J.-H. Lu et al., "Berberine ameliorates $\beta$-amyloid pathology, gliosis, and cognitive impairment in an Alzheimer's disease transgenic mouse model," Neurobiology of Aging, vol. 33, no. 12, pp. 2903-2919, 2012.

[34] S. S. K. Durairajan, A. Iyaswamy, S. G. Shetty et al., "HuanglianJie-Du-Tang reduces memory impairments and $\beta$-amyloid plaques in a triple transgenic mouse model of Alzheimer's disease," Scientific Reports, vol. 7, article 6238, 2017.

[35] Y. Ye, C. Huang, L. Jiang et al., "Huanglian-Jie-Du-Tang extract protects against chronic brain injury after focal cerebral ischemia via hypoxia-inducible-factor- $1 \alpha$-regulated vascular endothelial growth factor signaling in mice," Biological \& Pharmaceutical Bulletin, vol. 35, no. 3, pp. 355-401, 2012.

[36] O. Nakagawasai, F. Yamadera, K. Iwasaki et al., "Effect of kamiuntan-to on the impairment of learning and memory induced by thiamine-deficient feeding in mice," Neuroscience, vol. 125, no. 1, pp. 233-241, 2004.

[37] J. G. Hong, D. H. Kim, S. J. Park et al., "The memoryenhancing effects of Kami-ondam-tang in mice," Journal of Ethnopharmacology, vol. 137, no. 1, pp. 251-256, 2011.

[38] Z. L. Ren and P. P. Zuo, "Neural regeneration: role of traditional Chinese medicine in neurological diseases treatment," Journal of Pharmacological Sciences, vol. 120, no. 3, pp. 139-145, 2012.

[39] J. Kou, D. Zhu, and Y. Yan, "Neuroprotective effects of the aqueous extract of the Chinese medicine Danggui-Shaoyao-san on aged mice," Journal of Ethnopharmacology, vol. 97, no. 2, pp. 313-318, 2005.

[40] H. Yang, S. Wen, G. Zhang et al., "Effects of Chinese herbal medicine Fuzhisan on autologous neural stem cells in the brain of SAMP-8 mice," Experimental Gerontology, vol. 46, pp. 628636, 2011. 


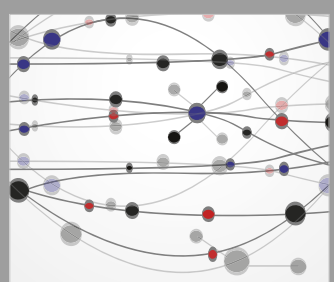

The Scientific World Journal
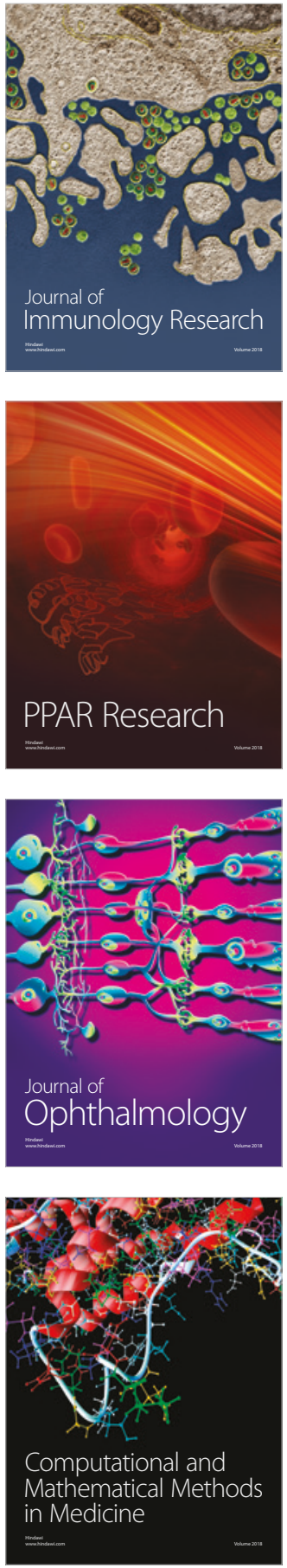

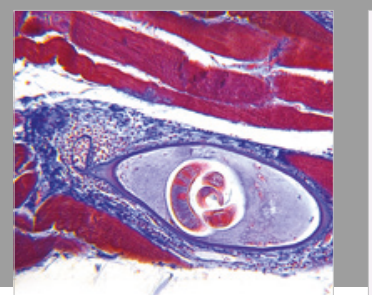

Gastroenterology Research and Practice

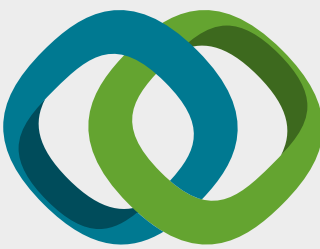

\section{Hindawi}

Submit your manuscripts at

www.hindawi.com
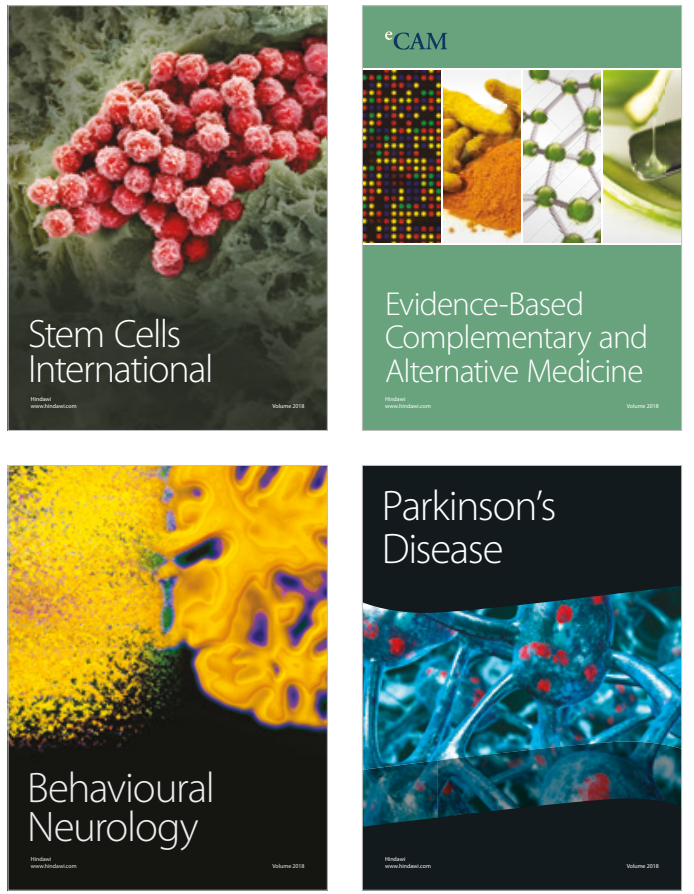

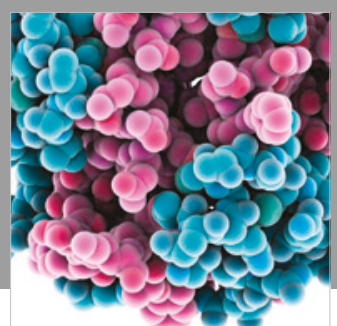

ournal of

Diabetes Research

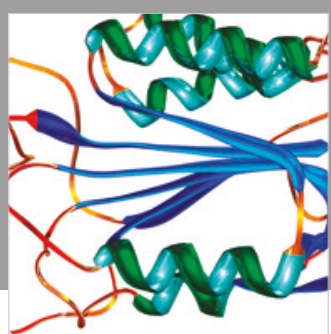

Disease Markers
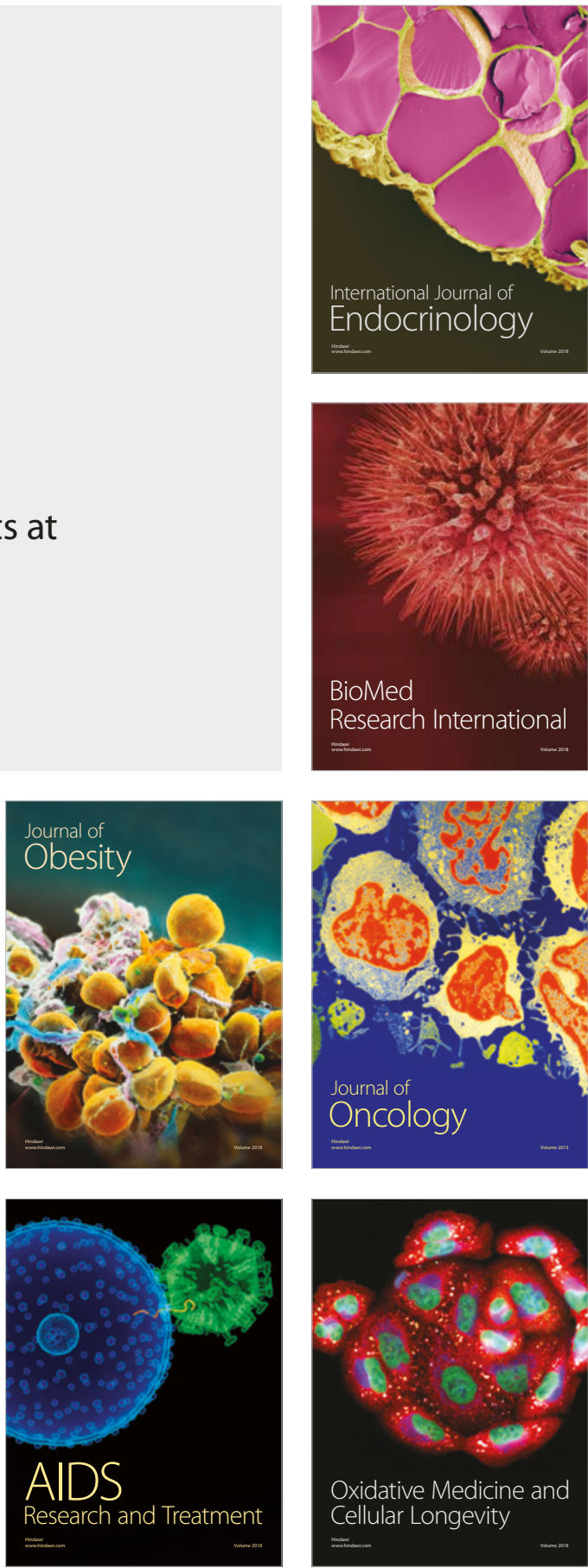